ARTICLE

Received 13 Aug 2014 | Accepted 11 Feb 2015 | Published 25 Mar 2015 DOI 10.1038/ncomms7626

\title{
Generation and expansion of highly pure motor neuron progenitors from human pluripotent stem cells
}

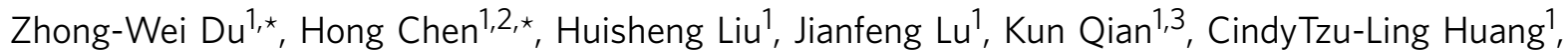
Xiaofen Zhong ${ }^{1}$, Frank Fan ${ }^{4} \&$ Su-Chun Zhang ${ }^{1,5}$

Human pluripotent stem cells (hPSCs) have opened new opportunities for understanding human development, modelling disease processes and developing new therapeutics. However, these applications are hindered by the low efficiency and heterogeneity of cell types, such as motorneurons (MNs), differentiated from hPSCs as well as our inability to maintain the potency of lineage-committed progenitors. Here by using a combination of small molecules that regulate multiple signalling pathways, we develop a method to guide human embryonic stem cells to a near-pure population (>95\%) of motor neuron progenitors (MNPs) in 12 days, and an enriched population ( $>90 \%$ ) of functionally mature MNs in an additional 16 days. More importantly, the MNPs can be expanded for at least five passages so that a single MNP can be amplified to $1 \times 10^{4}$. This method is reproducible in human-induced pluripotent stem cells and is applied to model MN-degenerative diseases and in proof-ofprinciple drug-screening assays.

\footnotetext{
${ }^{1}$ Waisman Center, University of Wisconsin, Madison, Wisconsin 53705, USA. ${ }^{2}$ Department of Rehabilitation Medicine, Tongji Hospital, Tongji Medical College, Huazhong University of Science and Technology, Wuhan 430030, China. ${ }^{3}$ Reproductive Medicine Center, Tongji Hospital, Tongji Medical College, Huazhong University of Science and Technology, Wuhan 430030, China. ${ }^{4}$ Promega Corporation, Madison, Wisconsin 53711, USA. ${ }^{5}$ Department of Neuroscience and Department of Neurology, School of Medicine and Public Health, University of Wisconsin, Madison, Wisconsin 53705, USA. ${ }^{*}$ These authors contributed equally to this work. Correspondence and requests for materials should be addressed to Z.W.D. (email: zhongweidu@wisc.edu) or to S.C.Z. (email: zhang@waisman.wisc.edu).
} 
uman pluripotent stem cells (PSCs), including embryonic stem cells (ESCs) and induced pluripotent stem cells (iPSCs), offer a new model system to explore early human development and dissect disease processes, as well as an opportunity to devise therapeutics ${ }^{1-3}$. A critical requirement for achieving these potentials is directed differentiation of hPSCs to target cell types. Substantial progress has been made in guiding hPSCs to major cell lineages, including blood, cardiac and neural cells $^{4-6}$. Nevertheless, generation of highly pure cellular populations in large quantities, which are often necessary for biochemical analysis, disease modelling and clinical application, has not been readily achieved. In particular, it is often desired to obtain functionally specialized subtypes of cells from hPSCs; however, these populations represent only a tiny fraction of the cells in a normal tissue/organ of our body. Such a need poses critical challenges to the stem cell field.

Spinal motor neurons (MNs) are a highly specialized type of neurons that reside in the ventral horns and project axons to muscles to control their movement. Degeneration of MNs is implicated in a number of devastating diseases, including spinal muscular atrophy (SMA), amyotrophic lateral sclerosis (ALS), Charcot-Marie-Tooth and poliomyelitis disease. The above disease iPSCs have been generated from patients and attempts have been made to identify disease-related phenotypes and to dissect out the underlying mechanisms before embarking on drug discovery ${ }^{7-10}$. However, these efforts are hindered by our inability to produce pure or highly enriched MNs with consistent quality. A number of protocols have been developed, including neural progenitor induction followed by neural patterning by retinoic acid (RA) and sonic hedgehog $(\mathrm{SHH})^{11,12}$, neural progenitor induction followed by genetic manipulations using adenovirusmediated gene delivery ${ }^{13}$ and differentiation of MNs with above methods followed by sorting with green fluorescent protein (GFP) labelling under the MNX1 (also known as HB9) promoter ${ }^{14}$. These differentiation protocols are tedious, time-consuming (1-2 months) and are of low efficiencies (30-70\%) unless by sorting (90\%). Furthermore, PSC-derived neurons in vitro, including MNs, are often immature, making it difficult to reveal disease phenotypes that are manifested in functionally mature cells. Hence, there is a critical need to develop new methods that enable generation of highly pure and functionally mature MNs with consistent quality and in a short time.

By using a combination of small molecules in a chemical defined neural medium, we have guided hPSCs to a near-pure population of $\mathrm{OLIG} 2^{+}$MNPs in 12 days, and a highly enriched population of functionally mature MNs (>90\%) in another 16 days by the use of a Notch inhibitor. Furthermore, our approach enables expanding a single MNP to $1 \times 10^{4} \mathrm{MNPs}$, producing a large quantity $\left(5 \times 10^{5}\right)$ of consistent MNs from a single stem cell. MNs generated by this method display molecular phenotypes of SMA and ALS, and can be readily adapted to screening platforms, as illustrated by our luciferase-based axonal length assay using ALS patient MNs.

\section{Results}

Generation of highly pure MNPs by a small-molecule cocktail. Specification of MNPs follows serial and overlapping developmental steps: neural induction as well as caudalization and ventralization of neuroepithelial progenitors (NEPs) ${ }^{11,15}$. In the presence of small molecules, SB431542 (SB, inhibitor of activinnodal signalling, $2 \mu \mathrm{M})$ and DMH1 (DMH, inhibitor of bone morphogenetic protein signalling, $2 \mu \mathrm{M})^{16,17}$, hESCs (line H9) were induced to NEPs, with $77 \pm 9 \%$ of the total differentiated cells being $\mathrm{SOX}^{+}$(Fig. 1a,b). Activation of WNT by a small molecular agonist CHIR99021 (CHIR) has been shown to promote neural induction and potentially also neuroepithelial proliferation $^{18,19}$. Under the treatment of $3 \mu \mathrm{M}$ CHIR together with $2 \mu \mathrm{M} \mathrm{SB}+2 \mu \mathrm{M}$ DMH for 6 days, nearly all the differentiated cells were SOX $1^{+}$NEPs (>98\%; Fig. 1a,b). CHIR not only augmented the efficiency of neural induction but also increased the yield of SOX1 ${ }^{+}$NEPs by 2.8 -fold (Fig. 1b). Wnt activation (by CHIR) often induces a caudal fate of neural progenitors $^{20}$. Indeed, CHIR-induced NEPs showed a caudal identity with HOXA3 expression. In contrast, NEPs induced by $\mathrm{SB}+\mathrm{DMH}$ (without CHIR) exhibited a rostral identity with OTX2 expression (Fig. 1b). Therefore, treatment of $\mathrm{CHIR}+\mathrm{SB}+\mathrm{DMH}$ combines the steps of induction and caudalization of NEPs, representing a chemically defined, single-step method for obtaining homogenous caudal NEPs from hPSCs.

The next step is to specify OLIG2 ${ }^{+}$MNPs by mimicking the ventralization of NEPs in vivo. By exposing the CHIR $+\mathrm{SB}+$ DMH-induced caudal-like NEPs to RA $(0.1 \mu \mathrm{M})$ and Pur (Purmorphamine, SHH signalling agonist, $1 \mu \mathrm{M}$ ) for 6 days, which was identified in our previous study ${ }^{21}$, we obtained $81 \pm 9 \%$ OLIG2-expressing neural progenitors (Fig. 1c). However, $\sim 40 \% \mathrm{OLIG}^{+}{ }^{+}$cells co-expressed with $\mathrm{NKX} 2.2$, another ventral spinal cord marker (Fig. 1c). During neural development, OLIG2 and NKX2.2 are initially induced in a common pool of progenitors that ultimately segregate into unique territories, giving rise to distinct $\mathrm{OLIG} 2^{+}$MNPs and $\mathrm{NKX} 2.2^{+}$interneuron progenitors ${ }^{22}$. WNT signalling plays a critical role in this segregation $^{23}$. We thus added WNT agonist CHIR in combination with RA and Pur. Under the treatment of $\mathrm{CHIR}+\mathrm{RA}+\mathrm{Pur}$ for 6 days, NKX2.2 expression was completely repressed in the culture, but OLIG2-expressing population was also decreased to $62 \pm 5 \%$ (Fig. 1c). We reasoned that WNT signalling may elevate the threshold of $\mathrm{SHH}$ signalling necessary to induce OLIG2 expression. However, at the increased concentration, $\mathrm{SHH}$ agonist Pur became toxic to the NEPs. We thus took an alternative approach to decrease the threshold of $\mathrm{SHH}$ signalling by repressing the dorsalizing molecule of the spinal cord, BMP signalling. Addition of dual inhibitors SB and DMH in combination with CHIR + RA + Pur significantly increased $\mathrm{OLIG} 2^{+} / \mathrm{NKX} 2.2^{-}$cell population (Fig. 1c). By serial titration of Pur and CHIR in combination with RA, SB and DMH (Supplementary Fig. 1), we found that treatment with $1 \mu \mathrm{M}$ CHIR, $2 \mu \mathrm{M}$ SB, $2 \mu \mathrm{M}$ DMH, $0.1 \mu \mathrm{M}$ RA and $0.5 \mu \mathrm{M}$ Pur for 6 days resulted in a robust population of OLIG2 ${ }^{+}$MNPs $(95 \pm 3 \%$ ), among which few (<0.5\%) OLIG2/ NKX2.2 double-positive cells were found (Fig. 1c).

Our protocol for $\mathrm{OLIG} 2+\mathrm{MNP}$ specification is highly reproducible in multiple different hPSC lines, including normal iPSC line IMR90, ALS iPSC lines SOD1-D90A and SOD1-A4V, and SMA iPSC lines SMA13 and SMA232 (Fig. 1d). Under the treatment of $\mathrm{CHIR}+\mathrm{SB}+\mathrm{DMH}$ for 6 days and $\mathrm{CHIR}+\mathrm{SB}+$ $\mathrm{DMH}+\mathrm{RA}+$ Pur for another 6 days, all the hPSC lines generated more than $90 \%$ OLIG2 ${ }^{+}$MNPs. Thus, coordinated specification and patterning of neuroepithelia by small molecules lead to robust generation of pure population of region-specific MNPs.

MNPs can be expanded to large numbers. Developmentally, OLIG2 ${ }^{+}$MNPs are present transiently and they transition to other neuronal (interneuron) and glial (oligodendrocyte) lineages after generation of MNs. For cellular and biochemical analyses, it is crucial to expand the MNPs without losing their ability to produce MNs, which has not been achieved. Since the CHIR+ $\mathrm{SB}+\mathrm{DMH}+\mathrm{RA}+$ Pur condition is highly efficient in specifying and generating MNPs, we asked whether the condition could 
a

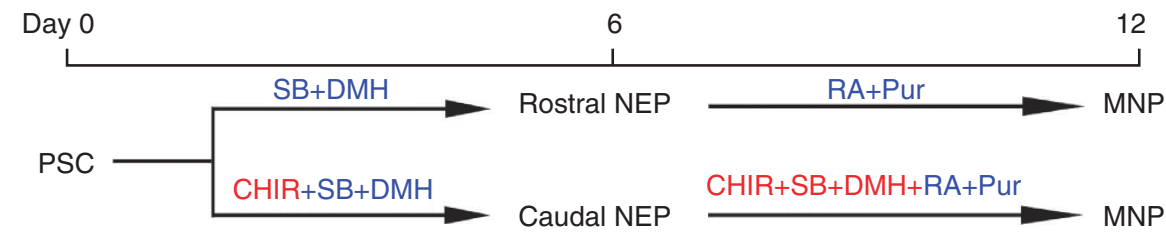

b $\mathrm{SB}+\mathrm{DMH}$ $\mathrm{CHIR}+\mathrm{SB}+\mathrm{DMH}$
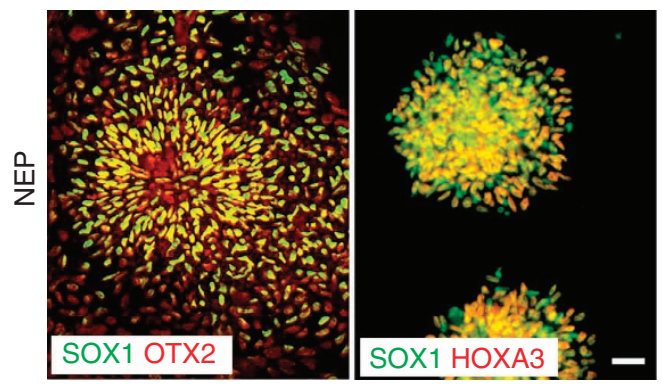

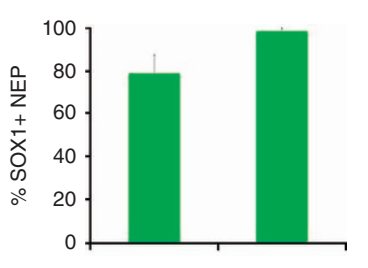

$\mathrm{SB}+\mathrm{DMH} \quad \mathrm{CHIR}+\mathrm{SB}+\mathrm{DMH}$
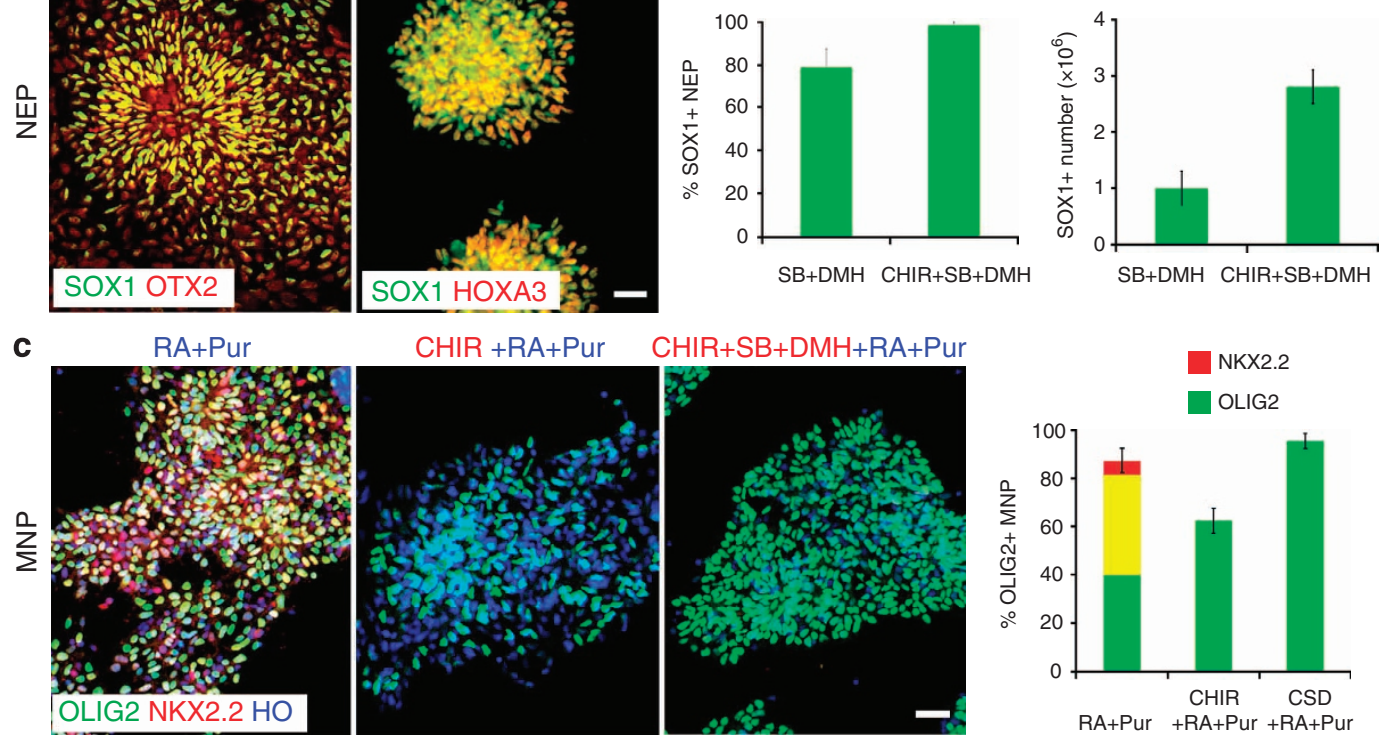

$\mathrm{CHIR}+\mathrm{RA}+\mathrm{Pur}$
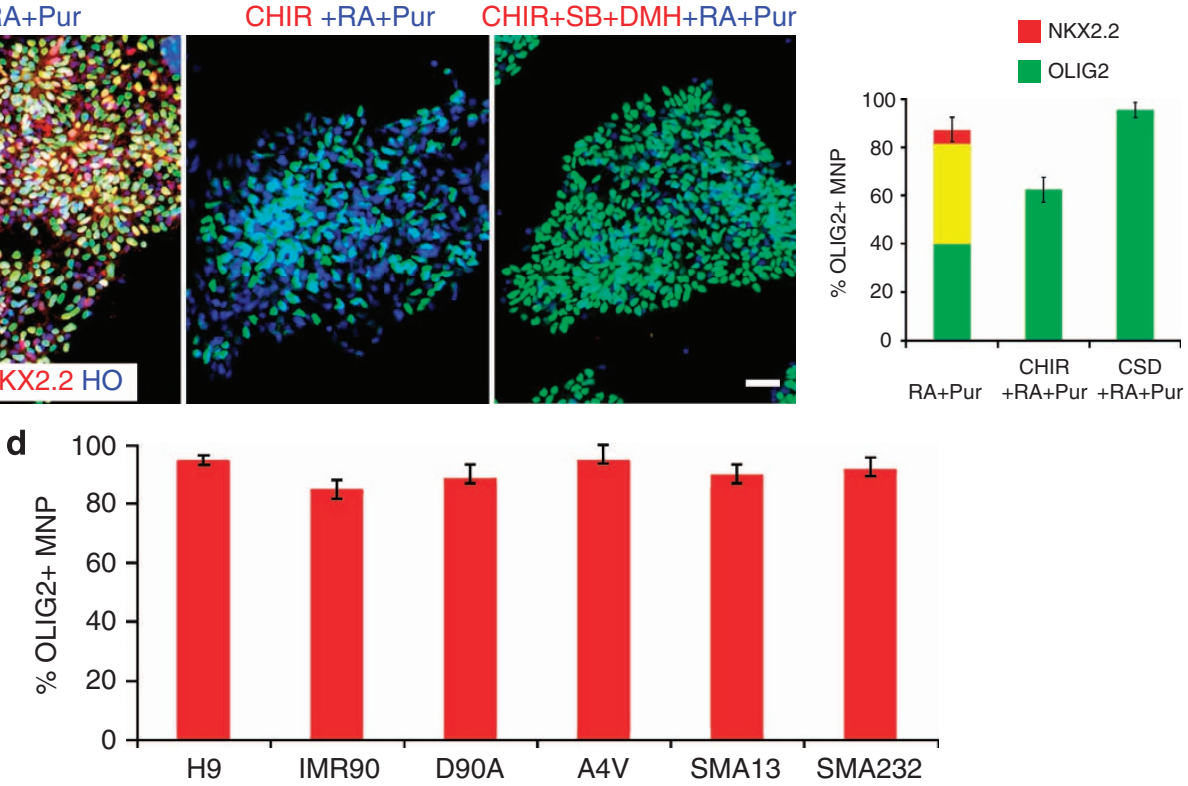

Figure 1 | Generation of highly pure population of MNPs from hPSCs. (a) Schematics showing the time course and small-molecule cocktail for hPSC differentiation into MNPs. (b) Representative images of SOX1 + NEPs after 6 days of culture in $\mathrm{CHIR}+\mathrm{SB}+\mathrm{DMH}$ versus SB+DMH condition. The regional identity (OTX2 + versus HOXA3 +) were stained. Scale bars, $50 \mu \mathrm{m}$. Quantification of SOX1 + NEP percentage and number is shown on right ( $>500$ cells from random fields were manually counted in each condition). The bar graph shows the mean \pm s.d. ( $n=3$ in each condition). (c) Representative images of pure MNPs on Day12 under different conditions, which express OLIG2 (green) but not NKX2.2 (red). Scale bars, $50 \mu$ m. Quantification of OLIG2 +, NKX2.2 + and OLIG2 + /NKX2.2 + cells is shown on right ( $>500$ cells from random fields were manually counted in each condition). The bar graph shows the mean \pm s.d. ( $n=3$ in each condition). (d) The efficiency of OLIG2 + MNP differentiation from multiple hPSC lines ( $>500$ cells from random fields were manually counted in each cell line). The bar graph shows the mean \pm s.d. ( $n=3$ in each cell line).

expand the OLIG2 ${ }^{+}$MNPs in a continuously dividing state. We first examined whether RA and Pur are required for maintaining OLIG2 expression. The MNPs were passaged weekly under the $\mathrm{CHIR}+\mathrm{SB}+\mathrm{DMH} 1$ condition with or without Pur or RA + Pur. After two passages, OLIG2 ${ }^{+}$MNPs were decreased to $35 \pm 5 \%$ in the control group (without Pur and RA), to $62 \pm 5 \%$ in Pur group, and a large population of NKX2.2 $2^{+}$cells appeared in these two groups. In the RA + Pur group, OLIG2 + MNPs were maintained at $91 \pm 3 \%$ with rare $N K X 2.2^{+}$cells (Fig. 2a). Therefore, RA and Pur are required for maintaining the identity of MNPs.

We then examined whether CHIR, SB and DMH are required for maintaining cell proliferation. The MNPs were passaged under the RA + Pur condition and divided into three groups: $\mathrm{SB}+\mathrm{DMH}$ treatment group, CHIR treatment group and $\mathrm{CHIR}+\mathrm{SB}+\mathrm{DMH}$ treatment group. After two passages, the cell population expressing Ki67, a cell proliferation marker, was at less than $8 \%, 81 \pm 6 \%$ and $92 \pm 4 \%$ in the $\mathrm{SB}+\mathrm{DMH}$ group, the $\mathrm{CHIR}$ group and the $\mathrm{CHIR}+\mathrm{SB}+\mathrm{DMH}$ group, respectively (Fig. 2b). Therefore, $\mathrm{CHIR}+\mathrm{SB}+\mathrm{DMH}$ are required for maintaining the proliferation of MNPs at the maximum level.

When the MNPs were expanded in the same media (CHIR+ $\mathrm{SB}+\mathrm{DMH}+\mathrm{RA}+$ Pur) for longer than two passages, the OLIG2-expressing MNP population decreased with a concomitant appearance of MNX1 expressing MNs, suggesting that some MNPs have exited cell cycle and differentiated to neurons. We reasoned that this is likely due to the neurogenic effect of RA. It was known that valproic acid (VPA), a histone deacetylase inhibitor, can repress neurogenesis by indirectly activating Notch signalling 24 . We thus added VPA to the culture system. Under this culture condition, the MNPs were expanded for at least five 


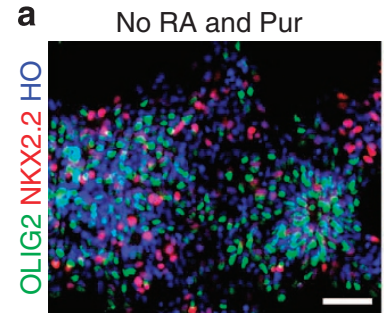

b

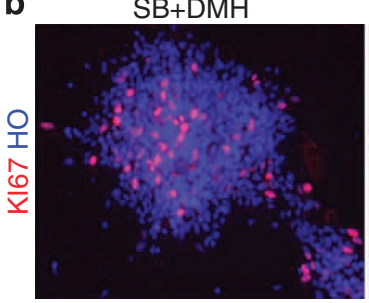

C

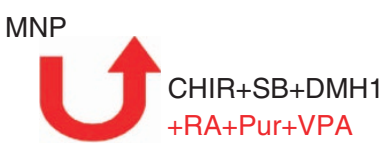

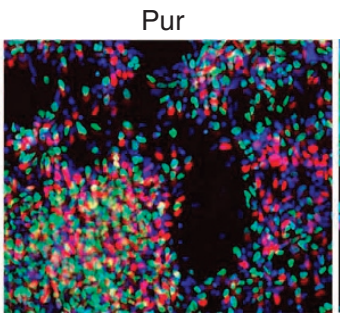

$\mathrm{CHIR}$

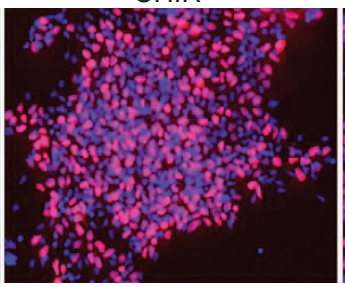

d

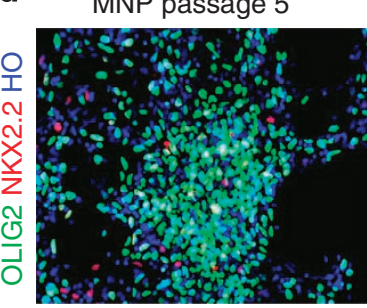

RA+Pur

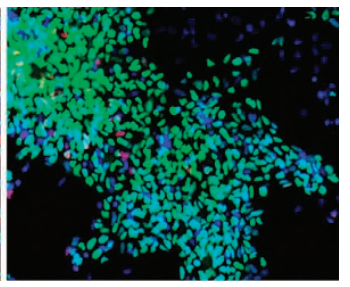

$\mathrm{CHIR}+\mathrm{SB}+\mathrm{DMH}$

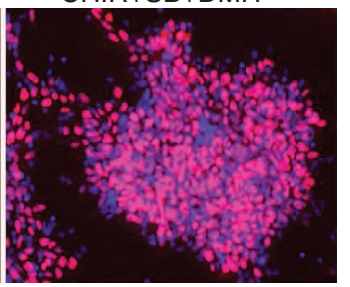

e

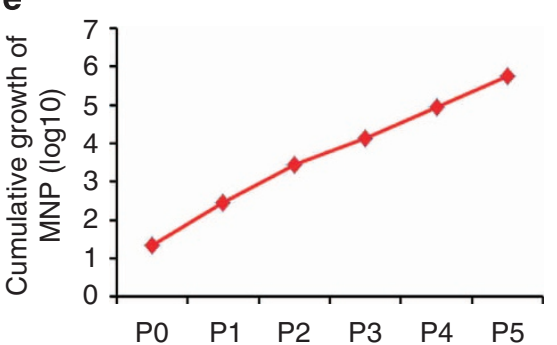

Figure 2 | Expansion of OLIG2 ${ }^{+}$MNPs. (a) Representative images of pure OLIG2 + (green)/NKX2.2+ (red) MNPs maintained under different conditions. Scale bars, $50 \mu \mathrm{m}$. Quantification of OLIG2 +/NKX2.2 - cells is shown on right ( $>500$ cells from random fields were manually counted in each condition). The bar graph shows the mean \pm s.d. ( $n=3$ in each condition). (b) Representative images of Ki67+ (red) proliferating progenitors maintained under different conditions. Scale bars, $50 \mu \mathrm{m}$. Quantification of Ki67+ ${ }^{+}$cells is shown on right ( $>500$ cells from random fields were manually counted in each condition). The bar graph shows the mean \pm s.d. ( $n=3$ in each condition). (c) Schematics showing the expansion of MNPs with the combination of small molecules. (d) Representative images of MNPs expanded for at least five passages, yet maintained the OLIG2 (green) expression. (e) Cumulative hPSC-derived MNP counts over five passages (passages denoted p1-p5). One six-well of cells were manually counted in each passage, and total cell numbers were calculated by time passage ratio.

passages, yet maintained OLIG2 expression at $82 \pm 9 \%$ (Fig. 2d). Further culturing under this condition resulted in a gradual decrease in $\mathrm{OLIG} 2^{+}$cell population and increase in NKX2.2 ${ }^{+}$ cell population, suggesting a need of alternative strategy for an even longer-term expansion. Nevertheless, continual expansion of MNPs for five passages allows amplification of a single MNP to $1 \times 10^{4} \mathrm{MNPs}$, translating to the generation of $>5 \times 10^{5} \mathrm{MNPs}$ from a single hPSC (Fig. 2e). Furthermore, these MNPs can be frozen and thawed under regular condition with over $90 \%$ recovery.

MNPs differentiate into an enriched population of functional MNs. To determine the differentiation of expanded MNPs, we withdrew $\mathrm{CHIR}+\mathrm{SB}+\mathrm{DMH}$, increased $\mathrm{RA}$ concentration $(0.5 \mu \mathrm{M})$ and reduced Pur $(0.1 \mu \mathrm{M})$. After 6 days, nearly all the MNPs differentiated into MNs, as evidenced by expression of MNX1 (90 $\pm 9 \%)$ or ISL1 (95 $\pm 3 \%$; Fig. 3a,b). Further culture on Matrigel or astrocyte feeders for 2 weeks resulted in generation of more mature MNs that expressed CHAT, although the $C H A T^{+}$ $\mathrm{MN}$ population $(47 \pm 9 \%)$ was substantially lower than the $M N X 1^{+}$MNPs. We reasoned that the lower population of $\mathrm{CHAT}^{+}$mature MNs may be due to proliferation of the small number of neural precursors and their subsequent differentiation to other neuronal types via lateral inhibition of NOTCH signalling 25 . To overcome this inefficiency of $\mathrm{MN}$ maturation, we applied Compound E (Cpd E), a NOTCH signalling inhibitor in the $\mathrm{MN}$ culture. Cpd E treatment for 10 days resulted in a near homogenous $\mathrm{MAP}^{+}$mature neuronal cultures without any proliferating cells $\left(\mathrm{Ki}_{6}{ }^{+}\right)$, and $\sim 91 \pm 6 \%$ of $\mathrm{MAP} 2^{+}$neurons expressed CHAT (Fig. 3a,b). These CHAT + MNs were electrophysiologically active, as defined by their ability to elicit action potentials in response to depolarizing current injection in current-clamp recordings (Supplementary Fig. 2). Therefore, Cpd E not only increases the mature $\mathrm{MN}$ population but also substantially shortens the maturation process.

To determine whether the Cpd E-accelerated MNs are functional, we co-cultured the MNs with differentiated myotubes from mouse $\mathrm{C} 2 \mathrm{C} 12$ cells. After 10 days of co-culture, we observed aggregated $\mathrm{BTX}^{+}$acetylcholine receptors on myotubes and their overlapping with $\mathrm{CHAT}^{+}$neurites (Fig. 3c), suggesting the formation of neuromuscular junctions. To study the ability of MNs to project axons towards the muscle targets, Cpd E-treated MNs were transplanted in ovo into the lesion neural tube of chicken embryos at $\mathrm{HH}$ stage15-16. Transplanted embryos showed successful engraftment of human MNs (as marked by GFP expression) into the ventral horn (Fig. 3d). Importantly, we observed GFP-labelled human $\mathrm{MN}$ axons $\left(\mathrm{CHAT}^{+}\right)$projected ventrally through the ventral roots and along the peripheral nerves of the host (Fig. 3d'). These data indicate that mature MNs generated by the Cpd E treatment exhibit proper functions.

Use of enriched MNs for disease modelling and screening. Most neurodegenerative diseases, such as SMA and ALS, preferentially affect one type of neurons such as MNs. Genetically linked 
a

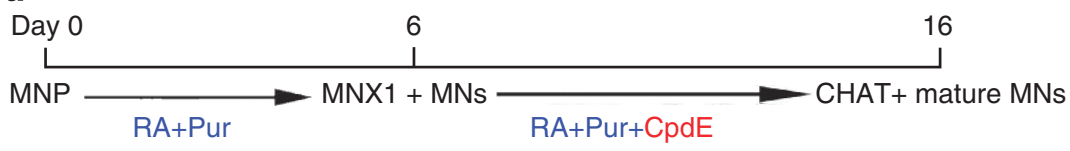

b MNX1 TuJ1 HO

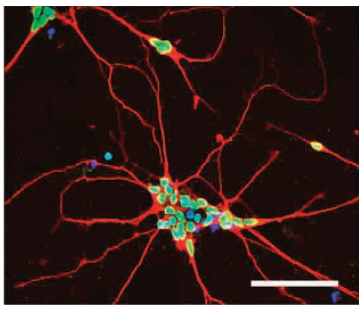

ISL1 TuJ1 HO

MAP2 CHAT
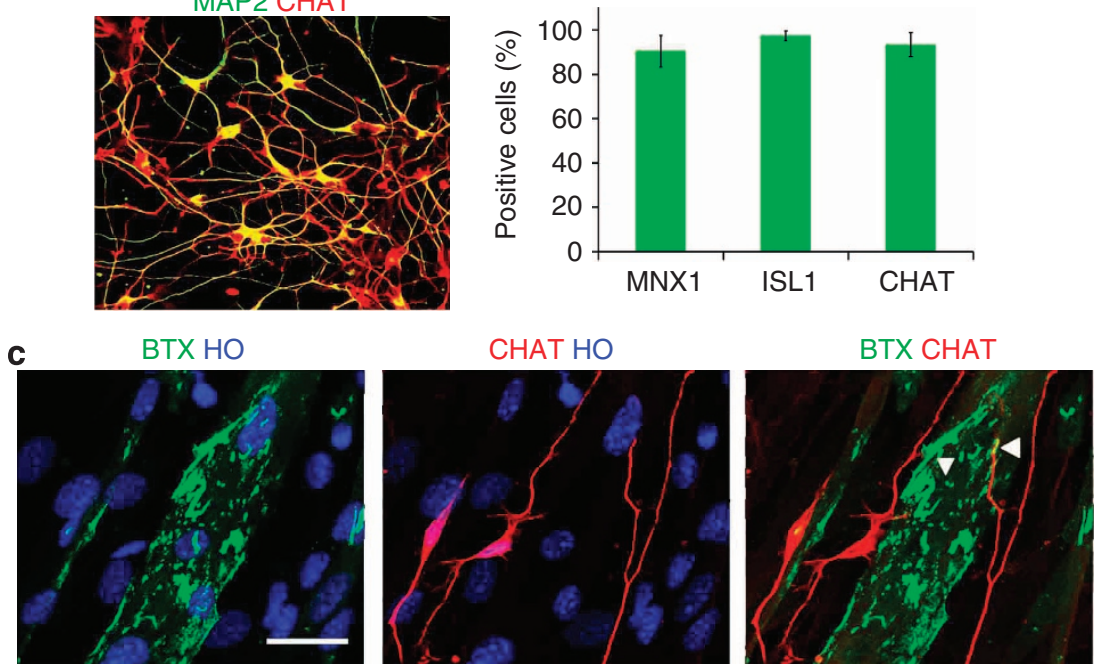

d

GFP HO

d'

GFP HO

GFP CHAT
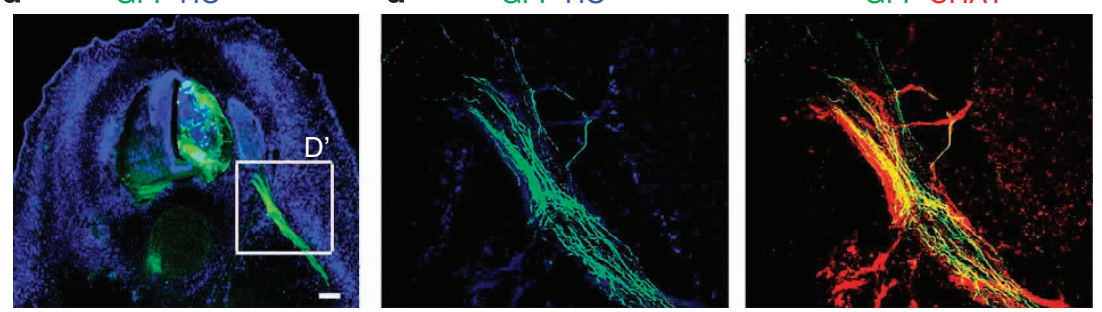

Figure 3 | MNPs differentiate into enriched functional MNs. (a) Schematics showing the time course and small-molecule cocktail for MNP differentiation into mature MNs ( $>500$ cells from random fields were manually counted in each condition). The bar graph shows the mean \pm s.d. ( $n=3$ in group). (b) Representative images of MNs showing $\mathrm{MNX}^{+}, \mathrm{ISL1}^{+}$(green) and $\mathrm{CHAT}^{+}$(red) on. Scale bars, $50 \mu \mathrm{m}$. Quantification of $\mathrm{MNX1}^{+}, \mathrm{ISL1}{ }^{+}$and $\mathrm{CHAT}{ }^{+}$ is shown (c) MNs, stained with the CHAT antibody (red), formed neuromuscular junctions, labelled with bungarotoxin (BTX, green), when co-cultured with myotubes. Scale bars, $100 \mu \mathrm{m}$. (d) Representative image of xenotransplantation of GFP-labelled human MNs into a developing chicken embryo. Scale bars, $50 \mu \mathrm{m}$. ( $\left.\mathbf{d}^{\prime}\right)$ Magnification of the field showing that human MN axons (GFP ${ }^{+} / \mathrm{CHAT}^{+}$) projected ventrally through the ventral roots.

disorders, including SMA and ALS, may lead to changes in gene dosage at less than $50 \%$. Hence, it will be technically difficult to discern changes in gene expression if the population of disease target cells is not highly enriched. To determine the utility of the MNs generated with the above method, we measured the expression of genes that are known to be altered in SMA and ALS. In this analysis, we generated spinal non-MNs from the same iPSCs as a control by replacing Pur with Cyclopamine to block SHH signalling (Supplementary Fig. 3a). Under RA and Cyclopamine treatment for 6 days, the induced spinal neural precursors were void of OLIG2 expression, and the differentiated neurons were void of MNX1 and CHAT expression, but with GABA ( $\gamma$-aminobutyric acid) expression (Supplementary Fig. 3b,c). Using these highly enriched MNs and GABA neurons, we found that the mRNA of survival motor neuron (SMN) was decreased in both MNs and GABA neurons that were derived from SMA patients as compared with those from non-SMA iPSCs (Fig. 4a). This is consistent with the fact that $S M N$ mutations affect all cell types. Interestingly, we found that MNs exhibited even lower ( $38 \pm 4 \%)$ SMN than that in GABA neurons $(60 \pm 6 \%$; Fig. 4a), again consistent with a previous report that MNs express markedly lower levels of full-length SMN transcripts from the SMN2 gene than do other cells in the spinal cord ${ }^{26}$. Similarly, in ALS caused by mutations in the superoxide dismutase (SOD1) gene, MNs display neurofilament (NF) aggregation that is attributed to the decreased level of light polypeptide neurofilament $(N E F L)^{9}$, we found a $45 \pm 4 \%$ reduction of $N E F L$ mRNA in D90A MNs, but not GABA neurons, when compared 

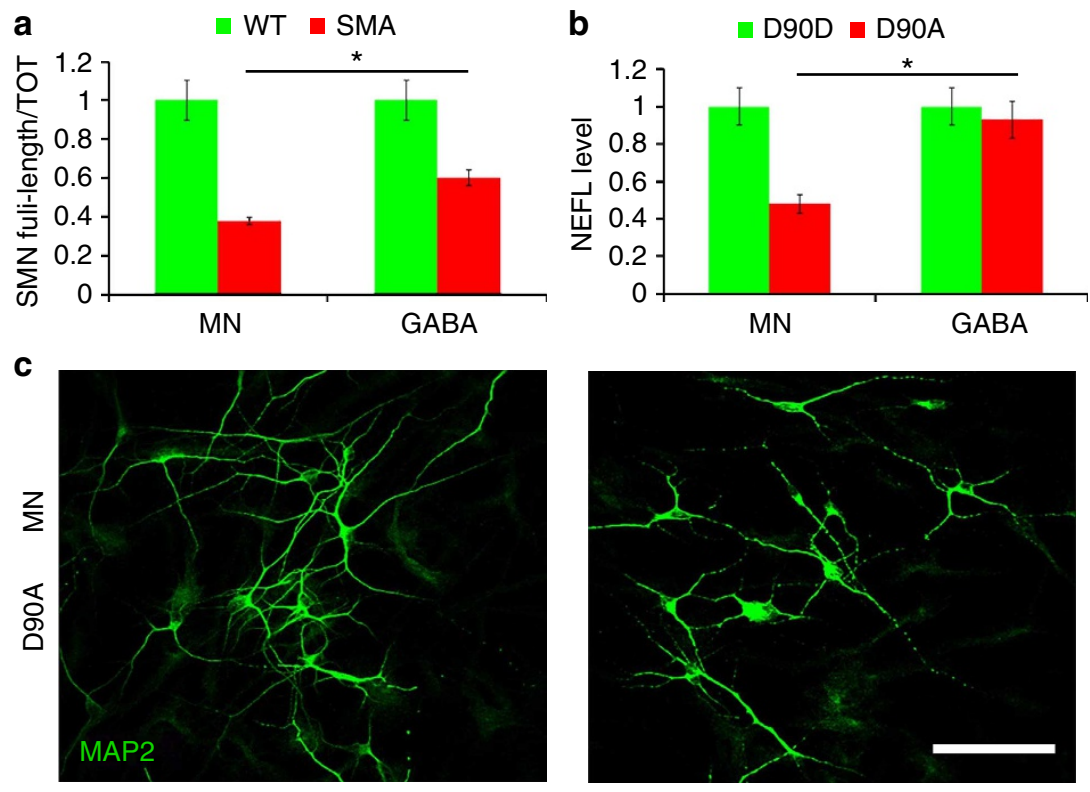

D90D astrocyte

D90A astrocyte

d

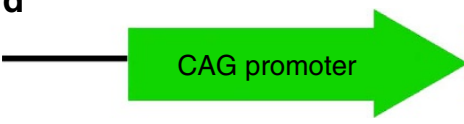

\section{Synaptophysin Nluc}
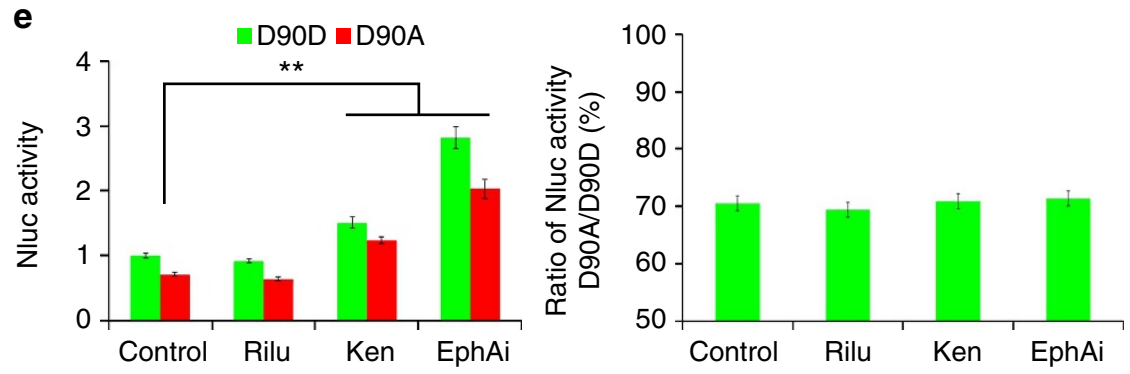

Figure 4 | Enriched MNs for disease modelling and screening. (a) The qPCR quantification of the ratio of full-length SMN versus total SMN in wild-type (WT) and SMA disease MNs, GABA neurons. The bar graph shows the mean \pm s.e.m. $\left({ }^{\star} P<0.05, t\right.$-test, $n=3$ in each group). (b) The qPCR quantification of the NEFL mRNA level in ALS (D90A) and corrected (D90D) MNs, GABA neurons. The bar graph shows the mean \pm s.e.m. $\left({ }^{\star} P<0.05, t\right.$-test, $n=3$ in each group). (c) Representative image of ALS (D90A) MNs when culturing on ALS (D90A) astrocytes and corrected (D90D) astrocytes, which showed neurite fragmentation and reduced neurite length. Scale bars, $50 \mu \mathrm{m}$. (d) Schematics of SYP-Nluc reporter. (e) Quantification of Nluc activity (left panel) and ratio (right panel) of SYP-Nluc reporter MNs on ALS (D90A) and corrected (D90D) astrocytes, when comparing between the control, Riluzole (Rilu), Kenpaullone (Ken) and EphA inhibitor (EphAi) groups. The bar graph shows the mean \pm s.e.m. ( ${ }^{\star} P<0.01$, Tukey's test, $n=8$ in each group).

with genetically corrected (D90D) MNs and GABA neurons (Fig. 4b). Together, these data indicate that the enriched MNs generated from patient iPSCs using our new method enable identification of disease-related phenotypes.

Our ability to generate large quantity of consistent MNs offers an opportunity for building high-throughput screening platforms for $\mathrm{MN}$ diseases. In ALS, astrocytes enhance disease progression by promoting axonal degeneration and MN death ${ }^{27-29}$. Indeed, when ALS iPSC-derived MNs were grown on top of ALS (D90A SOD1) or genetically corrected (D90D SOD1) astrocytes (Supplementary Fig. 4) in a medium that lacks neurotrophic factors, MNs began to show neurite fragmentation with reduced neurite length on D90A but not D90D astrocytes on Day 10 (Fig. 4c). To enable automated measurement of neurite length for high-throughput screening, we established a reporter iPSC line (from D90A SOD1) with a luciferase reporter NanoLuc (Nluc) fused with SYNAPTOPHYSIN (SYP; Fig. 4d), a synaptic vesicle glycoprotein, which targets the Nluc reporter to the axonal membrane, not the cytoplasm ${ }^{30}$. We first established the linear relationship between Nluc expression and $\mathrm{MN}$ numbers by measuring luciferase activity of cultures 10 days after plating different numbers of SYP-Nluc-expressing MNs (1,250, 2,500, $5,000,10,000$ and 20,000 cells) on astrocytes (Supplementary Fig. 5a). We then tested whether the Nluc reporter activity is correlated with the reduced axonal length. Same numbers of SYPNluc-expressing MNs were plated on D90D and D90A astrocytes, and the Nluc activity was detected on Day 10. The Nluc activity on D90A astrocytes was significantly decreased to $70.5 \pm 2.7 \%$, comparing with the D90D astrocyte group (Supplementary Fig. 5b). Next, we exposed the co-cultures to three compounds, Riluzole (Rilu) ${ }^{31}$, the only approved drug for ALS, as well as Kenpaullone (Ken) and EphA inhibitor (EphAi) that are known to rescue axonal degeneration in ALS cell models ${ }^{32,33}$. Riluzole had no effect on Nluc activity. Ken and EphAi increased the Nluc activity to 1.5- and 2.8-fold, respectively, in both D90A and D90D groups (Fig. 4e), indicating that they increased axonal length. However, Ken and EphAi did not specifically rescue the axonal length of MNs induced by ALS astrocytes, as the ratio of Nluc 
activity on $\mathrm{D} 90 \mathrm{~A}$ astrocytes versus $\mathrm{D} 90 \mathrm{D}$ astrocytes remained at $70 \%$ (Fig. 4e). These results provide a proof-of-principle for the use of our enriched, patient-derived MNs for drug screening and suggest its potential for identifying disease-specific targets.

\section{Discussion}

We have developed a strategy for guiding hPSCs to a near-pure population of $\mathrm{OLIG} 2^{+}$MNPs in 12 days by coordinating signalling pathways using small molecules, and subsequently a highly enriched population of functionally mature MNs ( $>90 \%)$ in another 16 days by the use of a Notch inhibitor. Furthermore, we have devised a method to expand a single MNP to $1 \times 10^{4}$ MNPs, enabling production of a large quantity $\left(5 \times 10^{5}\right)$ of consistent MNs from a single stem cell. Our approach enables presentation of disease phenotypes and building of screening platforms, as illustrated by our luciferase-based axonal length assay using ALS patient MNs.

Compared with previous methods, our method has two critical improvements. The first is the application of WNT agonist during MNP differentiation. WNT signalling is a more efficient pathway to caudalize neural progenitors ${ }^{20}$. Thus, the combination of WNT activator with dual SMAD inhibitors induced homogenous caudal NEPs from hPSCs. Most importantly, WNT signalling plays a critical role in MNP specification. All previous methods used RA and SHH to induce OLIG2 ${ }^{+}$MNPs without examining other ventral spinal markers, especially co-expression of NKX2.2. We showed here that RA and SHH condition generated mixed ventral progenitors with a large population of cells that co-express OLIG2 and NKX2.2. During spinal cord development, OLIG2 and NKX2.2 are initially induced in a common pool of progenitors that ultimately segregate into unique territories, giving rise to distinct $\mathrm{OLIG2}^{+}$MNPs (pMN domain) and NKX2.2 ${ }^{+}$ interneuron progenitors $(\mathrm{p} 3 \text { domain })^{22}$. Without segregation, NKX2.2 could interfere with the differentiation of OLIG2 ${ }^{+}$ progenitors to $\mathrm{MNX1}^{+} \mathrm{MNs}$, which is one of the reasons why some previous methods induced a high percentage of OLIG2 ${ }^{+}$ progenitors, but ended with a small population of $M N X 1^{+}$MNs. WNT signalling was reported to selectively oppose SHHmediated induction of NKX2.2, but have little effect on OLIG2, and thereby establish their distinct expression domains in cooperation with graded SHH signalling ${ }^{23}$. As WNT elevates the strength of $\mathrm{SHH}$ signalling to induce OLIG2 expression, the two inhibitors of dorsalizing BMP signalling were also included. Therefore, our method of combining with small molecules regulating WNT, SHH, RA and BMP signallings closely mimics the cooperation of these signallings in the spinal cord development in vivo to specify the region-specific OLIG2 ${ }^{+}$ MNPs. Recently Maury et al. ${ }^{34}$ also applied WNT agonist CHIR in the generation of MNs from hPSCs. However, the treatment condition under which CHIR was applied is different, and the NKX2.2-expressing cell population was not examined. It will be interesting to compare two approaches in specifying MNPs.

The second improvement is the application of a NOTCH inhibitor during $\mathrm{MN}$ maturation. Lateral inhibition mediated by NOTCH signalling is an intrinsic mechanism to guide orderly transition of mitotically active precursors into different types of post-mitotic neurons and glia at different stages ${ }^{25}$. The treatment with $\mathrm{NOTCH}$ inhibitor Cpd E in our method synchronizes the differentiation of $\mathrm{OLIG2}^{+}$MNPs to generate homogenous mature MNs without mixing with any other neural cells. With these two improvements, our method robustly generates almost homogenous mature MNs, which exhibit functional properties, including formation of neuromuscular junctions when cocultured with skeletal muscle cells and projection of axons towards muscles when grafted into the developing chick spinal cord. More significantly, the MNs derived from disease iPSCs by our method exhibit the MN-specific molecular phenotypes, including downregulation of full-length SMN in SMA and downregulation of the NEFL level in ALS, which would be nearly impossible to detect with previous methods that only generate a small population of $\mathrm{MN}$ in the mixed culture.

A large quantity of consistent target cells, such as mature MNs, is necessary for high-throughput screening. In general, lineagecommitted progenitors can be expanded, but quickly lose their differentiation potency. For example, $\mathrm{OLIG2}^{+}$MNPs can be expanded with fibroblast growth factor and/or epidermal growth factor, but quickly lose the potency of MN differentiation into two passages. Several recent reports described the expansion of neural progenitors with small molecules of WNT and/or SHH signallings ${ }^{18,35}$. However, as shown in this study (Fig. 2a), their ability to maintain $\mathrm{MN}$ potential is still significantly diminished during cell passages compared with our method. Our method can expand MNPs for at least five passages to amplify a single MNP to $1 \times 10^{4} \mathrm{MNPs}$, or generate $5 \times 10^{5} \mathrm{MNs}$ from single hPSC. This provides a sufficient cell source for high-throughput drug screening, as shown in our screening platform for MN axonal degeneration. In summary, our new method enables the generation of large quantities of MNs with consistency and high purity, providing a basis for modelling $\mathrm{MN}$ diseases in vitro and for drug discovery.

\section{Methods}

hPSCs. The human PSC lines used in this study are listed in Supplementary Table 1. Fibroblasts from a 50-year-old female ALS patient carrying the D90A SOD1 mutation (ND29149, Coriell Institute, coriell.org), a 3-year-old male SMA patient (GM03813, Coriell Institute) and a 7-month-old SMA patient (GM00232 Coriell Institute) were reprogrammed using the non-integrating Sendai virus as described $^{36}$ to established iPSC lines ALS-D90A, SMA13 and SMA232. The D90D iPSC line was established by correcting the D90A SOD1 mutation in ALS-D90A lines using TALEN technology ${ }^{9}$. A4V SOD1 mutant ALS iPSC line, established with retrovirus, was obtained from Coriell (ND35671). Human ESC line H9 (WA09 line, NIH registry 0046) and normal iPSC line IMR90-4 were obtained from WiCell. All the PSCs were cultured on irradiated mouse embryonic fibroblasts as described in the standard protocol http://www.wicell.org.

MNP specification and MN differentiation. To generate MNPs, hPSCs were dissociated with Dispase $\left(1 \mathrm{mg} \mathrm{ml}^{-1}\right)$ and split 1:6 on irradiated mouse embryonic fibroblasts or Matrigel-coated plates. On the following day, the PSC medium was replaced with a chemically defined neural medium, including DMEM/F12,

Neurobasal medium at 1:1, $0.5 \times \mathrm{N} 2,0.5 \times \mathrm{B} 27,0.1 \mathrm{mM}$ ascorbic acid (Santa Cruz), $1 \times$ Glutamax and $1 \times$ penicillin/streptomycin (all others from Invitrogen). CHIR99021 ( $3 \mu \mathrm{M}$, Torcris), $2 \mu \mathrm{M}$ DMH1 (Torcris) and $2 \mu \mathrm{M}$ SB431542 (Stemgent) were added in the medium. The culture medium was changed every other day. Human PSCs maintained under this condition for 6 days were induced into NEP cells. The NEP cells were then dissociated with Dispase $\left(1 \mathrm{mg} \mathrm{ml}^{-1}\right)$ and split at 1:6 with the same medium described above. RA $(0.1 \mu \mathrm{M}$, Stemgent) and $0.5 \mu \mathrm{M}$ Pur (Stemgent) were added in combination with $1 \mu \mathrm{M}$ CHIR99021, $2 \mu \mathrm{M}$ DMH1 and $2 \mu \mathrm{M}$ SB431542. The medium was changed every other day. NEP cells maintained under this condition for 6 days differentiated into OLIG2 + MNPs. The $\mathrm{OLIG}^{+}{ }^{+} \mathrm{MNPs}$ were expanded with the same medium containing $3 \mu \mathrm{M}$ CHIR99021, $2 \mu \mathrm{M}$ DMH1, $2 \mu \mathrm{M}$ SB431542, $0.1 \mu \mathrm{M}$ RA, $0.5 \mu \mathrm{M}$ Pur and $0.5 \mathrm{mM}$ VPA (Stemgent), and split 1:6 once a week with Dispase $\left(1 \mathrm{mg} \mathrm{ml}^{-1}\right)$. OLIG2 ${ }^{+}$ MNPs were frozen with the regular frozen medium (DMEM/F12, 10\% fetal bovine serum and $10 \%$ DMSO) in liquid nitrogen, and cultured again in expansion medium after thawing.

To induce MN differentiation, OLIG2 ${ }^{+}$MNPs were dissociated with Dispase $\left(1 \mathrm{mg} \mathrm{ml}^{-1}\right)$ and cultured in suspension in the above neural medium with $0.5 \mu \mathrm{M}$ RA and $0.1 \mu \mathrm{M}$ Pur. The medium was changed every other day. OLIG2 ${ }^{+} \mathrm{MNPs}$ under this condition for 6 days differentiated into $\mathrm{MNX1}^{+}$MNs. The $\mathrm{MNX1}^{+}$ MNs were then dissociated with Accumax (eBioscience) into single cells and plated on Matrigel-coated plates or on astrocytes. The $M N X 1^{+}$MNs were cultured with $0.5 \mu \mathrm{M}$ RA, $0.1 \mu \mathrm{M}$ Pur and $0.1 \mu \mathrm{M}$ Compound E (Calbiochem) for 10 days to mature into $\mathrm{CHAT}^{+}$MNs. Insulin-like growth factor 1, brain-derived neurotrophic factor and ciliary neurotrophic factor (all from $\mathrm{R} \& \mathrm{D}, 10 \mathrm{ng} \mathrm{ml}^{-1}$ each) were added if MNs were plated at low density. For identifying MN disease phenotypes, SMA and ALS MNs were cultured without these neurotrophic factors. 
Functional analysis of mature MNs. Whole-cell patch-clamp recordings were performed on iPSC-derived $\mathrm{CHAT}^{+}$neurons on Day28 after iPSC differentiation. Briefly, neurons were held at $-70 \mathrm{mV}$ to record $\mathrm{Na}+/ \mathrm{K}+$ channel activities, and at $0 \mathrm{mV}$ to record spontaneous release. For recording action potentials, cells were held at $0 \mathrm{pA}$ with the current-clamp model and the steps of currents from $+0 \mathrm{pA}$ were injected into cells by the holding electrode. The bath solution consisted of

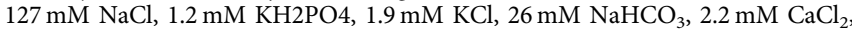
$1.4 \mathrm{mM} \mathrm{MgSO}_{4}, 10 \mathrm{mM}$ glucose, $290 \mathrm{mM}$ mOsm and $95 \% \mathrm{O}_{2} / 5 \% \mathrm{CO}_{2}$. Recording pipettes were filled with an intracellular solution containing $20 \mathrm{mM} \mathrm{KCl}$, $121 \mathrm{mMK}+-$ gluconate, $10 \mathrm{mM} \mathrm{Na}+-$ HEPES, $10 \mathrm{mM}$ BAPTA, $4 \mathrm{mM} \mathrm{Mg}_{2}+$ - ATP pH 7.2 and 290 mOsm. An Olympus BX51WI microscope was used to visualize neurons. A MultiClamp 700B amplifer (Axon Instruments, Molecular Devices, Sunnyvale, CA, USA) was used to investigate the voltage clamp and current-clamp recordings. Signals were filtered at $4 \mathrm{kHZ}$ and sampled at $100 \mathrm{kHz}$ using a Digidata $1322 \mathrm{~A}$ analogue-digital converter (Axon Instruments). Data were analysed with pClamp 9.0 (Axon Instruments). Capacitance and series resistance were compensated by $50-80 \%$ using an amplifier circuitry.

To examine neuromuscular junction formation, $\mathrm{C} 2 \mathrm{C} 12$ cells were seeded on Matrigel-coated plate in DMEM with 10\% FBS, and then were induced to form myotube by switching to DMEM containing $2 \%$ FBS. On Day $18 \mathrm{MNX}^{+}{ }^{+} \mathrm{MNs}$ derived from hPSCs were plated on myotubes and cultured in maturation condition for 7 days, after which the neuromuscular synapses were visualized using CHAT and BTX staining.

To perform transplantations, Day $18 \mathrm{MNX1}^{+} \mathrm{MN}$ spheres were triturated with a 1-ml pipette tip five to seven times and treated with Cpd E for $24 \mathrm{~h}$. Transplantation was performed as previously described ${ }^{15}$. Briefly, after a small suction, lesion at the prospective intraspinal site was created in a chick embryo at stage 15-18 at somites 15-20, and MN spheres were loaded into a hand-held microinjector and placed into the lesion. After 6 days, the chicks were killed, fixed with $4 \%$ paraformaldehyde for $2 \mathrm{~h}$ at $4{ }^{\circ} \mathrm{C}$ and neurite outgrowth was accessed by cutting $30-\mu \mathrm{m}$ sections of the spinal cord near the injected site.

qPCR analysis. Total RNAs were isolated with the RNeasy Plus Mini Kit (Qiagen) according to the manufacturer's instructions. For quantitative PCR (qPCR), cDNA was synthesized from $1 \mu \mathrm{g}$ total RNAs using iScript reverse transcription supermix (Bio-Rad). qPCR was performed using iTaq Universal SYBR Green Supermix (Bio-Rad). GAPDH gene was used as internal control to equalize cDNA. The following primers were used in this study: full-length SMN, forward-5'-CACCACC TCCCATATGTCCAGATT-3', reverse-5'-GAATGTGAGCACCTTCCTTCTTT- $3^{\prime}$; total SMN, forward-5'-ATGAGCTGTGAGAAGGGTGTTG-3', reverse-5' -TTGCC ACATACGCCTCACATAC- $3^{\prime}$; NEFL, forward-5' - TTTCACTCTTTGTGGTCC TCA-3' ${ }^{\prime}$, reverse-5'-AGACCCTGGAAATCGAAGC-3' ${ }^{\prime}$ GAPDH, forward-5'-CTCT CTGCTCCTCCTGTTCGAC- $3^{\prime}$ and reverse- $5^{\prime}$-TGAGCGATGTGGCTCGGCT- $3^{\prime}$.

Immunostaining and microscopy. Immunohistochemical staining was performed according to Zhang et al. (2001) $)^{37}$. The following primary antibodies were used: SOX1 (gIgG 1:1,000, R\&D), OTX2 (mIgG, 1:2,000, DSHB), HOXA3 (mIgG 1:1,000, R\&D), OLIG2 (rIgG 1:500, Chemicon), NKX2.2 (mIgG 1:100, DSHB), Ki67 (rIgG 1:200, Chemicon), MNX1 (mIgG 1:50, DSHB), ISL1 (mIgG 1:1,000, DSHB), TUJ1 (rIgG 1:5,000, Covance), CHAT (gIgG 1:300, Chemicon), MAP2 (mIgG 1:1,000, Chemicon), GABA (mIgG 1:1,000, Chemicon) and FoxP1 (rIgG, 1:1,000, Chemicon).

MN-astrocyte co-culture and luciferase assay. The luciferase reporter Nluc was obtained from Promega. The SYP-Nluc reporter iPSC line was established by inserting SYP-Nluc fusion reporter in the AAVS1 site by the TALEN technology ${ }^{38}$. Six-month astrocytes were differentiated from the isogenic iPSC lines D90D and D $90 \mathrm{~A}$ by the protocol established in our laboratory ${ }^{39}$. The astrocytes were plated at $1 \times 10^{4}$ cells per well in white 96-well plates (Greiner Bio-one) and cultured in the astrocyte medium (DMEM, 10\% FBS) for 7 days. The D90A MNX1 + MNs derived from SYP-Nluc reporter iPSC line were the plated at $1 \times 10^{4}$ cells per well on astrocytes and cultured in a nutrition-deficient medium (DMEM/F12, Neurobasal medium at $1: 1,1 \times \mathrm{N} 2,0.5 \mu \mathrm{M}$ RA and $0.1 \mu \mathrm{M}$ Compound E). For testing the compounds, Riluzole ( $50 \mu \mathrm{M}$, Torcris), Kenpaullone ( $5 \mu \mathrm{M}$, Tocris) and EphA inhibitor $(50 \mu \mathrm{M}$, Calbiochem) were added in the medium. After co-culturing for 10 days, the Nluc activity was detected by Nano-Glo Luciferase Assay (Promega) according to the manufacturer's instructions.

Statistical analyses. No statistical methods were used to predetermine sample sizes, but the samples sizes we used were consistent with those generally employed in the field. Comparisons between two groups were analysed by unpaired $t$-test with Welch's correction. Multiple comparisons were analysed by one-way analysis of variance followed by Tukey's multiple comparisons test. $P<0.05$ was considered to be statistically significant. Statistical processing was performed using the Microsoft Excel and GraphPad Prism Software.

\section{References}

1. Grskovic, M., Javaherian, A., Strulovici, B. \& Daley, G. Q. Induced pluripotent stem cells--opportunities for disease modelling and drug discovery. Nat. Rev. Drug Discov. 10, 915-929 (2011).

2. Han, S. S., Williams, L. A. \& Eggan, K. C. Constructing and deconstructing stem cell models of neurological disease. Neuron 70, 626-644 (2011).

3. Goldman, S. A., Nedergaard, M. \& Windrem, M. S. Glial progenitor cell-based treatment and modeling of neurological disease. Science 338, 491-495 (2012).

4. Ma, F. et al. Generation of functional erythrocytes from human embryonic stem cell-derived definitive hematopoiesis. Proc. Natl Acad. Sci. USA 105, 13087-13092 (2008).

5. Kattman, S. J. et al. Stage-specific optimization of activin/nodal and BMP signaling promotes cardiac differentiation of mouse and human pluripotent stem cell lines. Cell Stem Cell 8, 228-240 (2011).

6. Liu, H. \& Zhang, S. C. Specification of neuronal and glial subtypes from human pluripotent stem cells. Cell Mol. Life Sci. 68, 3995-4008 (2011).

7. Ebert, A. D. et al. Induced pluripotent stem cells from a spinal muscular atrophy patient. Nature 457, 277-280 (2009).

8. Egawa, N. et al. Drug screening for ALS using patient-specific induced pluripotent stem cells. Sci. Transl. Med. 4, 145 ra104 (2012).

9. Chen, H. et al. Modeling ALS with iPSCs reveals that mutant SOD1 misregulates neurofilament balance in motor neurons. Cell Stem Cell 14, 796-809 (2014).

10. Kiskinis, E. et al. Pathways disrupted in human ALS motor neurons identified through genetic correction of mutant SOD1. Cell Stem Cell 14, 781-795 (2014).

11. Li, X. J. et al. Specification of motoneurons from human embryonic stem cells. Nat. Biotechnol. 23, 215-221 (2005).

12. Qu, Q. et al. High-efficiency motor neuron differentiation from human pluripotent stem cells and the function of Islet-1. Nat. Commun. 5, 3449 (2014).

13. Hester, M. E. et al. Rapid and efficient generation of functional motor neurons from human pluripotent stem cells using gene delivered transcription factor codes. Mol. Ther. 19, 1905-1912 (2011).

14. Amoroso, M. W. et al. Accelerated high-yield generation of limb-innervating motor neurons from human stem cells. J. Neurosci. 33, 574-586 (2013).

15. Wichterle, H., Lieberam, I., Porter, J. A. \& Jessell, T. M. Directed differentiation of embryonic stem cells into motor neurons. Cell 110, 385-397 (2002).

16. Chambers, S. M. et al. Highly efficient neural conversion of human ES and iPS cells by dual inhibition of SMAD signaling. Nat. Biotechnol. 27, 275-280 (2009).

17. Neely, M. D. et al. DMH1, a highly selective small molecule BMP inhibitor promotes neurogenesis of hiPSCs: comparison of PAX6 and SOX1 expression during neural induction. ACS Chem. Neurosci. 3, 482-491 (2012).

18. Li, W. et al. Rapid induction and long-term self-renewal of primitive neural precursors from human embryonic stem cells by small molecule inhibitors. Proc. Natl Acad. Sci. USA 108, 8299-8304 (2011).

19. Lu, J. et al. Generation of integration-free and region-specific neural progenitors from primate fibroblasts. Cell Rep. 3, 1580-1591 (2013).

20. Xi, J. et al. Specification of midbrain dopamine neurons from primate pluripotent stem cells. Stem Cells 30, 1655-1663 (2012).

21. Li, X. J. et al. Directed differentiation of ventral spinal progenitors and motor neurons from human embryonic stem cells by small molecules. Stem Cells $\mathbf{2 6}$, 886-893 (2008).

22. Lee, S. K. \& Pfaff, S. L. Transcriptional networks regulating neuronal identity in the developing spinal cord. Nat. Neurosci. 4 (Suppl), 1183-1191 (2001).

23. Wang, H., Lei, Q., Oosterveen, T., Ericson, J. \& Matise, M. P. Tcf/Lef repressors differentially regulate Shh-Gli target gene activation thresholds to generate progenitor patterning in the developing CNS. Development 138, 3711-3721 (2011).

24. Stockhausen, M. T., Sjölund, J., Manetopoulos, C. \& Axelson, H. Effects of the histone deacetylase inhibitor valproic acid on Notch signalling in human neuroblastoma cells. Br. J. Cancer 92, 751-759 (2005).

25. Lewis, J. Notch signalling and the control of cell fate choices in vertebrates. Semin. Cell Dev. Biol. 9, 583-589 (1998).

26. Ruggiu, M. et al. A role for SMN exon 7 splicing in the selective vulnerability of motor neurons in spinal muscular atrophy. Mol. Cell Biol. 32, 126-138 (2012).

27. Di Giorgio, F. P., Carrasco, M. A., Siao, M. C., Maniatis, T. \& Eggan, K. Non-cell autonomous effect of glia on motor neurons in an embryonic stem cell-based ALS model. Nat. Neurosci. 10, 608-614 (2007).

28. Nagai, M. et al. Astrocytes expressing ALS-linked mutated SOD1 release factors selectively toxic to motor neurons. Nat. Neurosci. 10, 615-622 (2007).

29. Haidet-Phillips, A. M. et al. Astrocytes from familial and sporadic ALS patients are toxic to motor neurons. Nat. Biotechnol. 29, 824-828 (2011).

30. Nakata, T., Terada, S. \& Hirokawa, N. Visualization of the dynamics of synaptic vesicle and plasma membrane proteins in living axons. J. Cell Biol. 140, 659-674 (1998). 
31. Miller, R. G., Mitchell, J. D. \& Moore, D. H. Riluzole for amyotrophic lateral sclerosis (ALS)/motor neuron disease (MND). Cochrane Database Syst. Rev. 3, CD001447 (2012).

32. Yang, Y. M. et al. A small molecule screen in stem-cell-derived motor neurons identifies a kinase inhibitor as a candidate therapeutic for ALS. Cell Stem Cell 12, 713-726 (2013)

33. Van Hoecke, A. et al. EPHA4 is a disease modifier of amyotrophic lateral sclerosis in animal models and in humans. Nat. Med. 18, 1418-1422 (2012)

34. Maury, Y. et al. Combinatorial analysis of developmental cues efficiently converts human pluripotent stem cells into multiple neuronal subtypes. Nat. Biotechnol. 33, 89-96 (2015).

35. Reinhardt, P. et al. Derivation and expansion using only small molecules of human neural progenitors for neurodegenerative disease modeling. PLoS ONE 8, e59252 (2013).

36. Ban, H. et al. Efficient generation of transgene-free human induced pluripotent stem cells (iPSCs) by temperature-sensitive Sendai virus vectors. Proc. Natl. Acad. Sci. USA 108, 14234-14239 (2011).

37. Zhang, S. C. et al. Specification of transplantable astroglial subtypes from human pluripotent stem cells. Nat Biotechnol. 19, 1129-1133 (2001).

38. Qian, K. et al. A simple and efficient system for regulating gene expression in human pluripotent stem cells and derivatives. Stem Cells 32, 1230-1238 (2014).

39. Krencik, R. et al. Specification of transplantable astroglial subtypes from human pluripotent stem cells. Nat Biotechnol. 29, 528-534 (2011).

\section{Acknowledgements}

This study was supported by NIH-NINDS grants (NS045926 and NS064578 for S.C.Z., NS074189 and NS085689 for Z.W.D.) and in part by a core grant to the Waisman Center from the National Institute of Child Health and Human Development (P30 HD03352).

\section{Author contributions}

Z.W.D., H.C. and H.L. designed and performed experiments, analysed data and prepared figures. J.L. established ALS and SMA hiPSC lines. K.Q., C.T.-L.H., X.Z. and F.F. provide crucial data and reagents. Z.W.D. and S.Z. conceived the original idea, designed research and wrote the manuscript.

\section{Additional information}

Supplementary Information accompanies this paper at http://www.nature.com/ naturecommunications

Competing financial interests: The authors declare no competing financial interests.

Reprints and permission information is available online at http://npg.nature.com/ reprintsandpermissions/

How to cite this article: Du, Z.W. et al. Generation and expansion of highly pure motor neuron progenitors from human pluripotent stem cells. Nat. Commun. 6:6626 doi: $10.1038 /$ ncomms7626 (2015). 